\title{
Video Article \\ Imaging Amyloid Tissues Stained with Luminescent Conjugated Oligothiophenes by Hyperspectral Confocal Microscopy and Fluorescence Lifetime Imaging
}

\author{
Sofie Nyström ${ }^{1}$, Marcus Bäck ${ }^{1}$, K. Peter R. Nilsson ${ }^{1}$, Per Hammarström ${ }^{1}$ \\ ${ }^{1}$ IFM-Department of Chemistry, Linköping University
}

Correspondence to: Sofie Nyström at sofie.nystrom@liu.se, K. Peter R. Nilsson at petni@ifm.liu.se, Per Hammarström at perha@ifm.liu.se

URL: https://www.jove.com/video/56279

DOI: doi: $10.3791 / 56279$

Keywords: Biochemistry, Issue 128, Amyloid, hyperspectral imaging, confocal imaging, fluorescence life time imaging, luminescent conjugated oligothiophenes, histology.

Date Published: $10 / 20 / 2017$

Citation: Nyström, S., Bäck, M., Nilsson, K.P., Hammarström, P. Imaging Amyloid Tissues Stained with Luminescent Conjugated Oligothiophenes by Hyperspectral Confocal Microscopy and Fluorescence Lifetime Imaging. J. Vis. Exp. (128), e56279, doi:10.3791/56279 (2017).

\section{Abstract}

Proteins that deposit as amyloid in tissues throughout the body can be the cause or consequence of a large number of diseases. Among these we find neurodegenerative diseases such as Alzheimer's and Parkinson's disease afflicting primarily the central nervous system, and systemic amyloidosis where serum amyloid A, transthyretin and IgG light chains deposit as amyloid in liver, carpal tunnel, spleen, kidney, heart, and other peripheral tissues. Amyloid has been known and studied for more than a century, often using amyloid specific dyes such as Congo red and Thioflavin T (ThT) or Thioflavin (ThS). In this paper, we present heptamer-formyl thiophene acetic acid (hFTAA) as an example of recently developed complements to these dyes called luminescent conjugated oligothiophenes (LCOs). hFTAA is easy to use and is compatible with co-staining in immunofluorescence or with other cellular markers. Extensive research has proven that hFTAA detects a wider range of disease associated protein aggregates than conventional amyloid dyes. In addition, hFTAA can also be applied for optical assignment of distinct aggregated morphotypes to allow studies of amyloid fibril polymorphism. While the imaging methodology applied is optional, we here demonstrate hyperspectral imaging (HIS), laser scanning confocal microscopy and fluorescence lifetime imaging (FLIM). These examples show some of the imaging techniques where LCOs can be used as tools to gain more detailed knowledge of the formation and structural properties of amyloids. An important limitation to the technique is, as for all conventional optical microscopy techniques, the requirement for microscopic size of aggregates to allow detection. Furthermore, the aggregate should comprise a repetitive $\beta$-sheet structure to allow for hFTAA binding. Excessive fixation and/or epitope exposure that modify the aggregate structure or conformation can render poor hFTAA binding and hence pose limitations to accurate imaging.

\section{Video Link}

The video component of this article can be found at https://www.jove.com/video/56279/

\section{Introduction}

Deposition of amyloid in tissue is a pathologic hallmark in a number diseases such as Alzheimer's disease, Parkinson's disease, systemic amyloidosis, and prion diseases. Despite the prevalence of amyloid-related diseases, and the fact that close to 40 different proteins to date have been classified as amyloid precursors in human ${ }^{1}$, little is known about the relation between amyloid deposition and disease phenotype. Histology of samples from human patients has been used both for diagnostic and scientific purposes. A large number of animal models have been established to investigate the correlation between amyloid burden and behavior, life span, and a number of other phenotypic read outs of disease progression ${ }^{2,3}$. Large efforts are also being made in drug discovery and design to battle some of our most feared widespread diseases. However, the evaluation of the connection between genotype, phenotype, amyloid plaque load, and drug administration is not straight forward. The tools for staining and imaging of amyloid in tissue are often blunt and provide low resolution information on amyloid formation and structure.

Congo red birefringence, ThT , and ThS fluorescence are examples of classical methods for detecting and analyzing amyloid burden in tissue samples from patient biopsies and post mortem samples, and animal models of disease ${ }^{4}$. These techniques have been used for several decades (Congo red since the 1920s and ThT and derivatives since the 1960s), and although instrumentation has been refined and allows for detailed analysis, the staining procedures and analysis are still being performed in the same manner as almost a century ago.

In this paper, we describe the utilization of a highly sensitive novel amyloid dye, $\mathrm{hFTAA}^{5}$, that allows the detection of small immature protein deposits with high accuracy, as well as detection of mature amyloid. Compared to conventional dyes, hFTAA has been proven to detect a wider range of disease associated protein aggregates ${ }^{5,6,7}$. In addition, hFTAA can also be applied for optical assignment of distinct aggregated morphotypes ${ }^{8}$. Herein, we describe hFTAA staining and analysis of tissue from established animal models of prion disease and Amyloid- $\beta$ Precursor Protein (APP) transgenic mice designed to mimic plaque development in Alzheimer's disease ${ }^{9,10}$. We also show analysis of diagnostic and post mortem samples from patients suffering from systemic amyloidosis. The LCOs have intrinsic properties that enables them to report on conformational differences within one plaque; and by combining two LCOs with different properties regarding binding and fluorescence, the 
difference is even more pronounced ${ }^{11}$. An epifluorescence microscope equipped with long pass filters and a hyperspectral camera head is used to enable objective classification of spectral properties and recording of micrographs for spectral analysis. Confocal fluorescence microscopy with a tunable laser as the excitation source is used to evaluate the three-dimensional properties of an amyloid plaque in greater detail. The tunable laser enables collection of excitation spectrum and a step less selection of emission wavelengths on the microscope enables co-imaging of LCO fluorescence and immunofluorescence to determine co-localization of target protein and amyloid. FLIM offers unprecedented sensitivity to conformational differences imposed on the LCOs and reveals differences that might not be detected on the fluorescence emission spectra.

The main goals with the described staining method are to facilitate sensitive detection of small amyloid deposits and to characterize conformational polymorphism within amyloid deposits. This knowledge is important for the basic understanding of protein aggregation diseases.

\section{Protocol}

NOTE: LCO synthesis has been performed in our labs for more than a decade. By essentially applying protocols for electrophilic aromatic substitution, palladium catalyzed cross-coupling, amide coupling, and ester hydrolysis, we synthetically customize probes for different purposes ${ }^{5,12}$. After synthesis, characterization, and purification, the LCO product is lyophilized and stored at room temperature. Some of the probes (among them hFTAA) are now commercially available (see Table of Materials).

\section{LCO Staining Solution}

NOTE: If hFTAA is purchased from a commercial vendor, please follow the vendor's instructions instead of section 1.

1. Resuspend the lyophilized hFTAA in $2 \mathrm{mM} \mathrm{NaOH}$ to prepare a stock solution of $1 \mathrm{mg} / \mathrm{mL}$. Keep the stock in a glass vial at $4{ }^{\circ} \mathrm{C}$. The stock can be stored for one year.

2. On the day of staining, prepare a working solution by diluting the stock 1:10,000 in Phosphate-buffered saline (PBS).

\section{Preparation of Tissue Samples}

NOTE: Many tissue types can be imaged using hFTAA as an amyloid marker. See Figure 1 for examples. hFTAA is sensitive to aggregate conformation. The staining should hence preferably be performed on undisrupted aggregates with no epitope exposure. Optimal spectral quality is achieved if the tissue fixation is kept to a minimum. Hence fresh frozen material, gently fixed in ethanol at the time of staining, is preferred. However, it is possible to detect amyloid deposits also in tissue that has been fixed with e.g., formalin. hFTAA generally penetrates the tissue well. Select a specimen thickness that is compatible with the intended imaging technique.

1. If formalin fixed, parafinembedded sections are used, deparafinize in xylene over night. Dip the sections in consecutive baths of $99 \%$ ethanol, $70 \%$ ethanol, $\mathrm{dH}_{2} \mathrm{O}$, and PBS, 10 min in each. Allow the tissue sections to dry under ambient conditions.

Caution: Xylene is always handled in a chemical fume hood. Xylene and other organic solvents are harmful.

1. Thaw cryosections in room temperature. Fix the tissue sections in $10 \%$ formalin overnight and rehydrate by dipping them in consecutive baths of $99 \%$ ethanol, $70 \%$ ethanol, $\mathrm{dH}_{2} \mathrm{O}$, and PBS, 10 min in each. Allow the tissue sections to dry under ambient conditions.

2. Add droplets of the hFTAA working solution (approximately $200 \mu \mathrm{L}$ ) to the tissue sections to cover it. The droplet should stay in place by surface tension. Incubate for 30 min at room temperature for staining.

3. Rinse off the staining solution with $500 \mu \mathrm{L}$ PBS using a pipette and then immerse the slide in the PBS bath for 10 min. Allow the section to dry under ambient conditions.

4. Mount using the fluorescence mounting medium. Allow mounting medium to settle overnight.

NOTE: hFTAA staining can be performed in combination with other staining methods such as immunofluorescence, cell- or organelle specific markers, etc. To perform co-staining, run your complete staining protocol of choice and add hFTAA staining at the end, starting from step 2.2. See Figure 2 for examples. For immunofluorescence, preferably select a secondary antibody that is excited at $640 \mathrm{~nm}$ or higher. In this wavelength range, hFTAA does not absorb and hence cannot be excited and will not fluoresce. This ensures that no bleed-through is seen between hFTAA and antibody.

\section{Microscopy}

NOTE: Use a fluorescence microscope equipped with long pass filters. All settings below were used to generate the images in Figure 4. Adjustments may need to be made depending on the amyloid type and tissue sample. Although hFTAA bound to amyloid is stable towards bleaching, it is advisable to turn off the light source when the specimen is not examined or imaged.

1. HIS

NOTE: The experiment was performed using an epifluorescence microscope with long pass emission filters and a camera head for HIS (see Table of Materials).

1. Use a standard fluorescence microscope equipped with long pass filters and a hyperspectral camera. Make sure that the spectral camera is calibrated.

2. In the software, name the project, select "spectral image" and start acquisition.

3. Select the object of interest through the ocular using the 436-nm excitation filter and shift the light path to the camera.

4. In the Case Data Manager (CDM), select the sample type Spectral, label the sample, and press acquire. The acquisition window will open.

5. In the acquisition window "Spectral imaging", open the settings menu, select acquisition properties, and set the spectral range to 460 700 , the speed quality at maximum speed, and the measurement type at gas/laser/narrow filters. Close the dialog box. 
6. In the image menu, select live full. In the icons bar, turn fringes off. Select or size a region to image that has the peak memory value below $800 \mathrm{MB}$. Set the exposure time to a value that gives a total image brightness between 1,000 and 3,000. In the icons bar, press the colored camera. Acquisition will start. When image acquisition is complete, press Save in the "Acquire spectral image" dialog box and "New cell" in the case data manager dialog box.

7. From the CDM, open the collected image using the start analysis button. The data analysis window will open.

8. Spectral information can be collected from each pixel of the image by selecting ROls using the Spectral display dialog box. Choose "define" and select ROls from the relevant areas of the image.

9. Save the spectral data as a text file using the Lib button. The saved .txt file can be imported to any analysis software of choice. The .slb file can be used for analysis within the data analysis software. The hyperspectral cube data from the entire image can also be exported as a raw file, as "layers as tif", or as "layers in text format" for data and image analysis applications using external software.

2. Confocal microscopy

NOTE: The confocal microscope is equipped with a tunable laser as the excitation source. For all fluorescence emission experiments, set the laser intensity to $0.2 \%$ (corresponding to an average power of $3 \mu \mathrm{W}$ ), the pinhole to 1 airy unit, the frame size to $1,024 \mathrm{px} x 1,024 \mathrm{px}$, the scan speed as 7 and averaging over 16 scans, and the bit depth as 8 bit (see Table of Materials). These settings need to be adjusted for each individual confocal system, laser source, and sample type.

1. For the emission spectrum, collect the data using lambda mode and the excitation using argon laser set to $488 \mathrm{~nm}$. Collect emission between 503 and $687 \mathrm{~nm}$ using 22 channels in the 32 channel GASP detector. Set the gain to 755 .

2. To achieve single channel images, use the smart set up option. For FITC (green filter), set the gain to 750 and for Alexa 535 (red filter) set the gain to 845 .

3. To collect the excitation spectrum with the tunable laser, use excitation with $1 \mathrm{~nm}$ steps between 490 and $545 \mathrm{~nm}$ while collecting emission between 551 and $586 \mathrm{~nm}$. Set the laser intensity to $2 \%$ (corresponding to an average power of $30 \mu \mathrm{W}$ ) and the gain to 774 .

4. To collect Z-stack in the spectral mode, scan through the depth of the section in steps of $0.96 \mu \mathrm{m}$. The same excitation and emission settings as in step 3.2.1 can be used but set the gain to 730 .

3. FLIM

NOTE: The confocal microscope is equipped with a FLIM unit (see Table of Materials).

1. Set up the following parameters for the confocal microscope: Pinhole, 20; excitation wavelength, $490 \mathrm{~nm}$; laser intensity, $0.5 \%$ (corresponding to an average power of $7.5 \mu \mathrm{W}$ ). Use pulsed lasers at $40 \mathrm{MHz}$.

2. In the FLIM software, set up photon counting over $550 \mathrm{~nm}$. In the Display parameters window, follow the photon counting until the Max count is around 4,000 photon counts.

3. Save the file and export it as SPC image.

4. Fit the data to a 2-component exponential decay in the FLIM software. A fit that gives a $x^{2}<2$ is good. The value on the $y$-axis is the number of counts for the given lifetime.

5. Select a threshold for counts to include, e.g., 100. Color code by T1 and select lifetime range. The decay is dependent on the amyloid structure where the hFTAA is bound. Fluorescence lifetimes between 300 and 1,000 ps have been observed. Save the file.

6. Export the raw data by using the export options and save the desired data in a new folder.

\section{Representative Results}

Sensitive and selective staining of amyloid deposits from a large number of proteins in many different tissue types from both human patients and experimental animals is vital for clinical diagnostics as well as in scientific research. In this paper, we demonstrate how this can be achieved using LCOs, exemplified by hFTAA, for staining and multimodal imaging of amyloid from a selection of tissue types. Since the first publication of thiophene-based amyloid ligands over ten years ago ${ }^{13}$, amyloid deposits composed of a wide range of proteins in a variety of tissues have been imaged using LCOs ${ }^{6,7,11,14,15,16}$ (Figure 1). In combination with antibodies or other histological markers, the LCOs provide excellent tools for both diagnostic and scientific purposes (Figure 1a, Figure 2). With an appropriate selection of fluorescent markers, several fluorophores can be imaged on the same section (Figure 1a, hFTAA and DAPI, Figure 2a hFTAA in immunofluorescence setup). It is also possible to use consecutive sections for staining using brightfield and fluorescence (Figure $\mathbf{2 b}$, hFTAA and DAB antibody staining). Recently, hFTAA has been proven to be a highly promising complement to Congo red staining in clinical diagnostics ${ }^{7,15}$ (Figure 3).

Development of imaging techniques over the last decades has increased the need of amyloid dyes that can discriminate between minute, but at the same time profound differences in amyloid deposits in a quantitative manner. The conjugated system within the LCO provides it with a unique ability to report on variation in its mode of binding. Twisting of the molecule can lead to distortion in the binding angles in the conjugated system and impede the transport of electrons (Figure 4a). This in turn reflects on the fluorescent properties of the LCO both in excitation and emission wavelength and in emission lifetime. We use HIS in an upright fluorescence microscope equipped with long pass filters for emission. For excitation and emission spectra in confocal microscopy and FLIM, we use an LSM780 with pulsed laser. To exemplify the different techniques, we imaged one object (the beta amyloid (A $\beta$ ) plaque in an APP transgenic mouse) (Figure 4b-e). Hyperspectral fluorescence spectra (Figure $\mathbf{4 b}$ ) allows for the evaluation of spectral shifts and intensity variation within different regions of the plaque. Excitation spectrum in confocal microscopy (Figure 4c) can be used to determine the optimal excitation wavelength for downstream experiments, e.g., combination staining with several fluorophores. This method can also prove useful for detection of conformational differences in the binding mode of the LCO. Emission spectrum in confocal mode can be used to determine the fluorescence emission properties from hFTAA or from a combination of several fluorophores to evaluate colocalization in combination experiments with several LCOs or LCO and immunofluorescence combinations. The fluorescence lifetime of hFTAA is highly influenced by small variations in the binding mode of hFTAA. Hence FLIM is a powerful tool in discriminating between the differences imposed on hFTAA by the binding target (Figure 4d). This can be used for example in the discrimination between different prion strains ${ }^{8}$. Confocal imaging and processing Z-stacks into three-dimensional images is a useful tool for exploring the overall shape of an amyloid aggregate (Figure 4e). Again, the use of additional fluorophores can aid in understanding the composition of a deposit. 

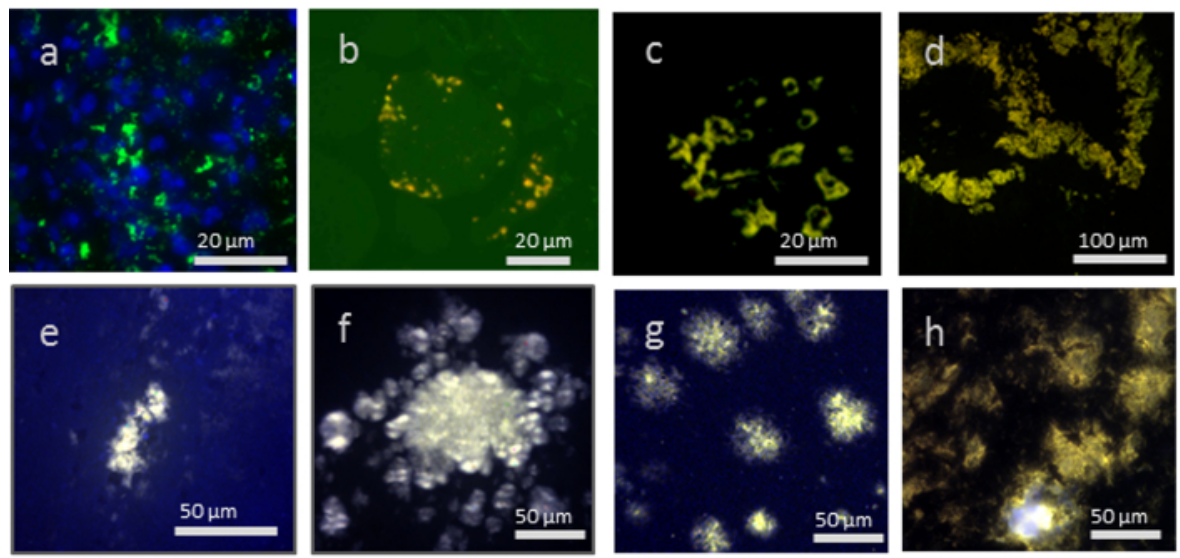

$\ddot{\mathrm{i}}$
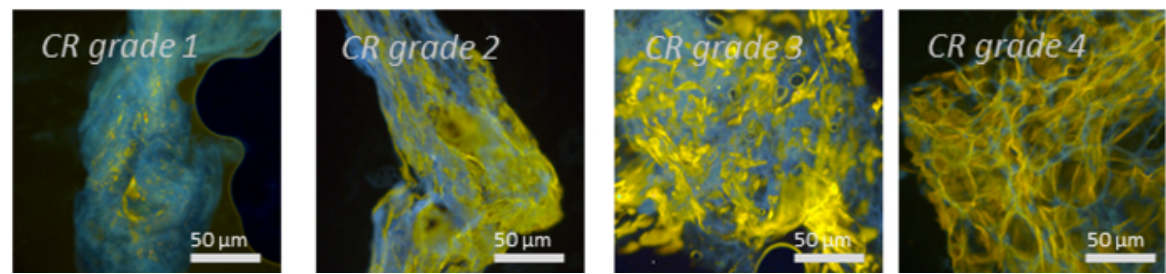

Figure 1: Various tissue types and proteins aggregates showing h-FTAA staining of: (a) Mallory-Denk bodies consisting of keratin aggregates in a liver (counterstained with DAPI), (b) p62-positive r inclusions in sporadic inclusion-body myositis (s-IBM) muscle tissue, (c) Amyloid of islet amyloid polypeptide in human pancreas, (d) Amyloid of Immunoglobulin light chain in human intestine, (e) Sheep scrapie (prions) in mouse brain, (f) Chronic wasting disease (prions) in mouse brain, (g) A $\beta$ plaques in APP23 mouse brain, (h) A $\beta$ pathology in APP/PS1 mouse brain, and (i) Fat biopsy smears from diagnostic samples of transthyretin amyloid in human patients graded 1 - 4 according to standard Congo red (CR) scoring. Yellow areas show hFTAA stained amyloid deposits and blue is autofluorescence from adipose tissue. The scale bar length is specified in each panel. Please click here to view a larger version of this figure.

a

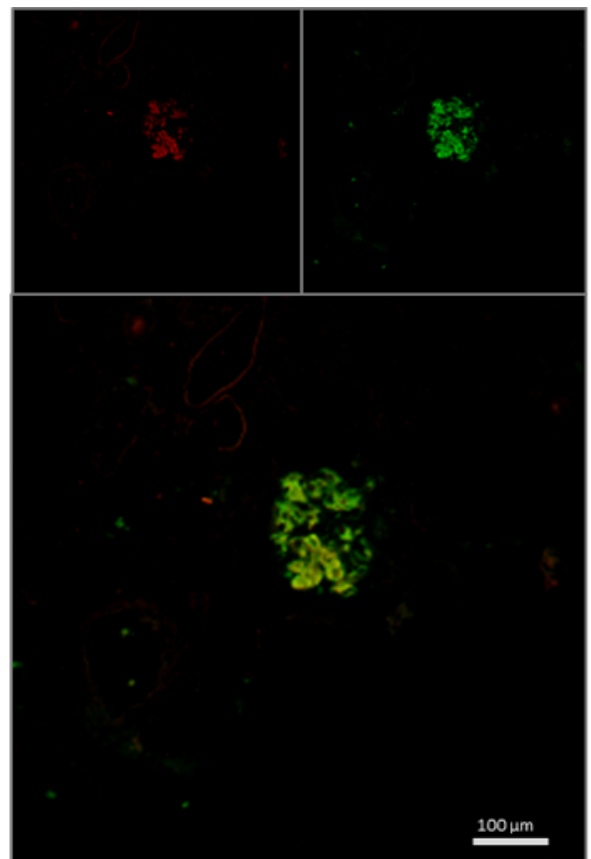

b

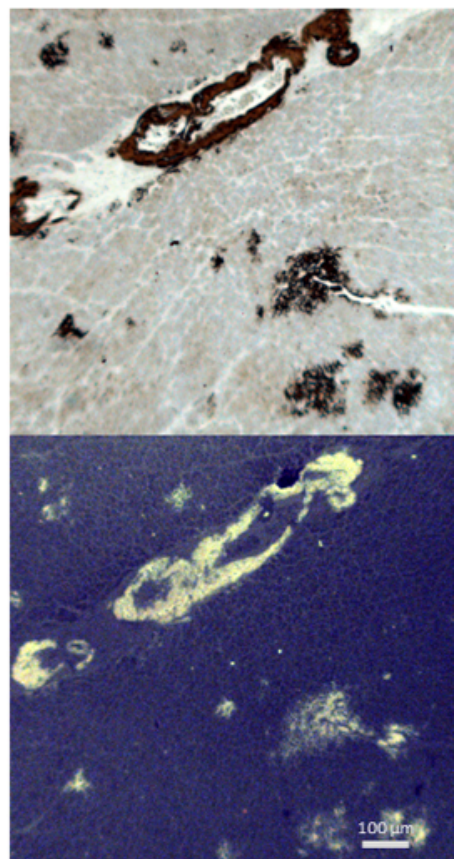

Figure 2: Examples of co-staining with antibodies. (a) Co-staining with anti-serum amyloid protein $A$ (AA) immunofluorescence and hFTAA of human AA amyloid on the same section. Upper left: AA antibody emission $640 \mathrm{~nm}$; upper right hFTAA at $488 \mathrm{~nm}$; bottom panel overlay image showing colocalization in yellow. (b) Antibody staining and hFTAA fluorescence on consecutive sections. Top panel: AA antibody DAB staining, bottom panel: hFTAA imaged with a longpass emission filter. Scale bar: $100 \mu \mathrm{m}$. Please click here to view a larger version of this figure. 
a

b

c

d

e

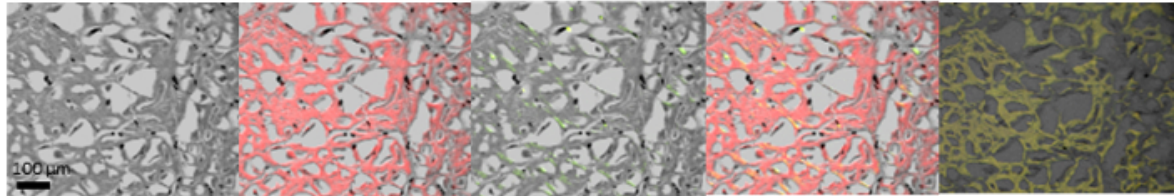

Figure 3: Comparing fluorescence with short pass filters on transthyretin amyloid in human heart stained with Mayer's hematoxylin/Congo red (a-d) and Mayer's hematoxylin/hFTAA (e). (a) Brightfield image, (b) Brightfield + fluorescence, (c) Brightfield + crossed polarizers, (d) Brightfield + fluorescence + crossed polarizers, (e) hFTAA fluorescence in $405 \mathrm{~nm}+480 \mathrm{~nm}$ excitation (consecutive sections to a-d). Scale bar: $100 \mu \mathrm{m}$. Please click here to view a larger version of this figure.

a

b
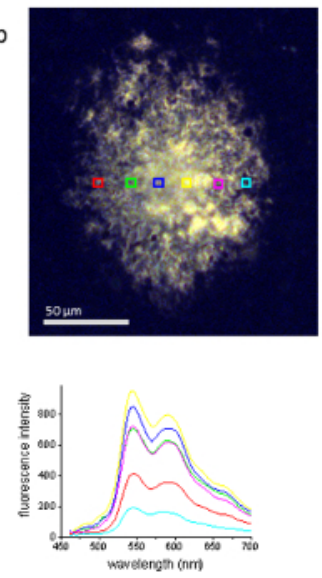

d
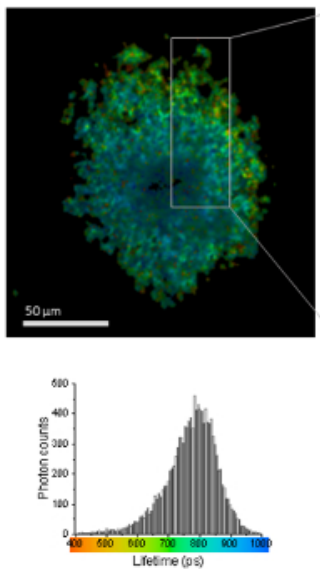
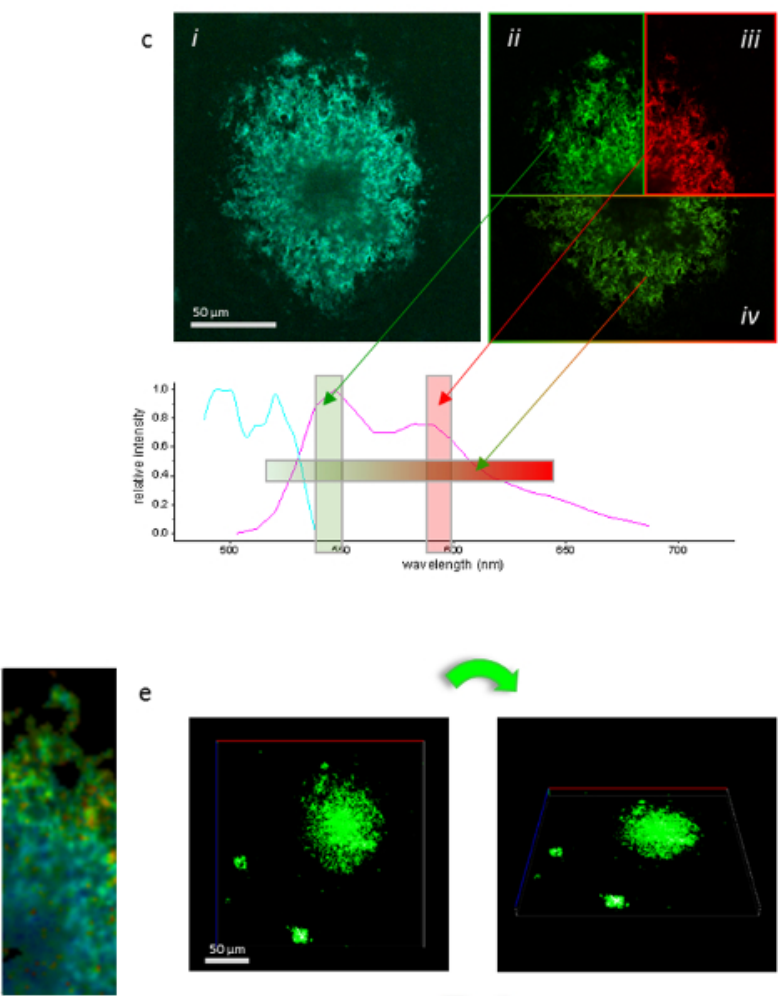

e
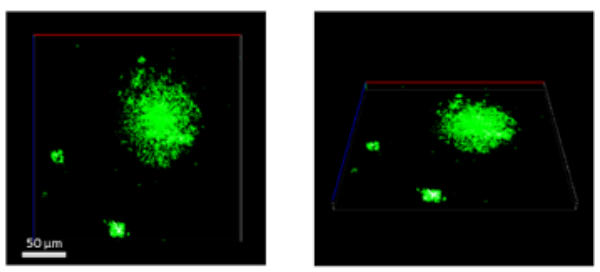

ㄴ.

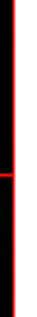


Discussion

Deposition of aberrantly folded proteins into amyloid is a key event of many disease processes. Extracellular amyloid plaques composed of $A \beta$ peptides and intracellular neurofibrillary tangles formed by hyperphosphorylated tau are found in the brain of Alzheimer's disease patients. A range of different proteins, e.g., transthyretin (TTR), Serum Amyloid A component (SAA), and IgG light chain misfold and manifest as amyloid deposits in tissues outside of the CNS. Although amyloid deposits have been known and studied for more than a century, we still lack detailed knowledge of how amyloid deposition is initiated at the molecular level and what can be done to prevent this process. For Alzheimer's disease, it is necessary to corroborate how the process of amyloidosis is associated with neurodegeneration. One key quest on the mission to treat amyloidosis is to develop novel fine-tuned tools for monitoring amyloid initiation, progression, and regression; hence targeted amyloid detection is key. In the long run, clinical diagnostics will benefit from increasing the sensitivity and selectivity of amyloid staining methods, ${ }^{7,15}$. Current challenges in this respect are tremendous and is a very active research area. One main issue for clinical development and trials is prognostic diagnosis. These diseases likely start well before symptoms appear, but to start with treatment there needs to be identification of the disease. Herein, sensitivity is a key aspect for novel methodologies. Furthermore, this issue is even more complex because some protein aggregates are toxic, some are protective, and some are neutral. Hence the ability to monitor specific aggregated morphotypes is essential since the existence of distinct aggregated species has been suggested to explain the heterogeneous phenotype reported for a diversity of neurodegenerative protein aggregation diseases. For instance, the prion protein is a classic example of how an identical primary sequence of amino acids can misfold into distinct aggregate morphotypes, which give rise to specific prion strains. Similar polymorphism has also been reported for the A $\beta$ peptide, $\alpha-$ synuclein, and tau. In this regard, LCOs have been shown to be outstanding tools for optical assignment of distinct aggregated morphotypes. Prion-strain-specific protein aggregates, protein deposits found in several types of systemic amyloidosis, as well as polymorphic $A \beta$ and tau aggregates have been distinguished due to the conformationally induced optical phenomenon observed from the LCOs.

Amyloid deposition is an event that takes place in tissues that are hard to penetrate with biochemical or biophysical methods of molecular precision. The possibility to probe events ex vivo, in vivo, and in vitro using the same LCO molecules sets the stage for unraveling events that occur in vivo using techniques that are only possible to apply on ex vivo or in vitro samples ${ }^{11,17}$. A recently reported high resolution structural model shows that the LCO pentameric FTAA (pFTAA) binds in a cavity formed by aligned side chains parallel with the fibril axis spanning 6 inregister parallel beta-strands ${ }^{18}$, demonstrating that it is possible to gain atomic resolution knowledge about the entities of the LCO target. In essence the binding cavity and binding mode of PFTAA is similar to Congo red ${ }^{19}$, dictated by a groove lined with repetitive positive charged Lys side-chains. The affinity of LCOs appear to be better compared to Congo red likely due to chain flexibility and strong van der Waals interactions of the sulfur atoms of the thiophene rings towards the hydrophobic cavity. The detection of prefibrillar species (before ThT responds) ${ }^{5,20}$ appears dependent on repetitive $\beta$ sheets, composed of in-register parallel-beta-strands, which for hFTAA being two thiophene units longer than pFTAA would span crossing of up to 8 beta-strands.

The staining protocol requires paying attention to trouble shooting at the following steps: (i) Fixation: Extensive fixation of tissue samples can disrupt the amyloid structure and limit the possibility to detect variation in fluorescence spectra induced by conformational distortion of the hFTAA molecule. Mild fixation of cryosections from fresh-frozen material is preferred to achieve optimal spectral resolution. However, hFTAA will stain and fluoresce amyloid in fixed tissue but with reduced efficacy and less spectral variation. (ii) Epitope exposure: Pre-treatment of tissue to achieve epitope exposure for antibody binding might occasionally reduce the ability for hFTAA to bind because of disrupted amyloid structure. If this is an issue, and epitope exposure is a crucial step in the antibody staining protocol, using consecutive sections for antibody and hFTAA, respectively can be considered. (iii) Overstaining: hFTAA is extremely sensitive. Working solutions should be kept at low nM concentration. Decrease the concentration of hFTAA if background staining is a problem. If elements that bind hFTAA are sparse in the tissue, an excess of hFTAA can aggregate and accumulate in the mounting medium. This is recognized as fluorescence that is not in the focus plane of the tissue itself. If this appears, immerse the mounted slide in PBS until the cover slip can be slid of the section, wash with PBS, and remount with fresh mounting medium and a new cover slip. (iv) Filter based imaging: This paper describes mostly spectrally resolved fluorescence imaging using long pass filters or multiple detection channels. hFTAA can also be monitored using short pass filters. Please be aware that this will reduce the contrast and abolish the possibilities to detect multiple colors.

Over the past years it has become evident that as research tools, fluorescent LCOs and in particular hFTAA show highly promising properties. Results have been demonstrated for a vast variety of proteins and disease states, ranging from hFTAA detection of aggregates inside cells (tau, inclusion body myositis), systemic amyloid of serum amyloid A, transthyretin, Immunoglobulin light chains (kappa and lambda) seminogelin 1, prion protein (including clinical samples) ${ }^{21}$, Islet amyloid polypeptide, and insulin ${ }^{7,15}$. A limiting factor for the implementation of hFTAA as a diagnostic tool is that fluorescence microscopy is not extensively used in routine amyloid pathology labs. Furthermore, HIS is not readily available in the clinic. However, hFTAA amyloid staining and fluorescence microscopy have been used in clinical labs in a filter based fluorescence microscope and should be considered as a complimentary diagnostic method. This was recently demonstrated on carpal tunnel biopsies with transthyretin amyloidosis using basic settings for fluorescence in an automated fashion ${ }^{22}$.

Furthermore, in addition to various proteins, hFTAA fluorescence recognizes a vast variety of fibril types and pre-fibrillar amyloid aggregates, e.g., pathway fibrillar species have been detected and characterized. In this publication, we have demonstrated one LCO on various sample types using three microscopy techniques. The use of controlled synthesis has also allowed for the development of LCOs for versatile detection of biomolecular targets, e.g., recording of interactions by surface plasmon resonance (SPR) ${ }^{23}$ and radiolabeling for positron emission tomography $(\mathrm{PET})^{24}$

To date, over 25 different LCOs with have been published. An increased use of LCOs for imaging of amyloid deposits will increase the knowledge and understanding of how these disease-associated aggregates assemble, disassemble, and interfere with the organs and individuals in which they reside. 


\section{Disclosures}

PH PN MB SN are minor shareholders in Ebba Biotech that commercializes hFTAA under the brand name Amytracker545.

\section{Acknowledgements}

The authors wish to thank Mikael Lindgren and Chanan Sluzny for advice on fluorescence microscopy, and Adriano Aguzzi, Johan Bijzet, Bouke Hazenberg, Frank Heppner, Mathias Jucker, Therese Klingstedt, Karin Magnusson, Christina Sigurdson, Daniel Sjölander, Christoph Röcken, Gunilla Westermark, Per Westermark, and Kurt Zatloukal for contributing tissue sections or micrographs displayed in this publication. The collection of presented data herein has been financed by contributions from Swedish Brain Foundation (Hjärnfonden), the Swedish Alzheimer association (Alzheimerfonden), the Swedish research council (VR), the Göran Gustafsson association, Georg \& Astrid Olsson, EU FP7 Health project LUPAS, and Linköping University.

\section{References}

1. Sipe, J. D., et al. Amyloid fibril proteins and amyloidosis: chemical identification and clinical classification International Society of Amyloidosis 2016 Nomenclature Guidelines. Amyloid. 23, 209-213 (2016).

2. Buxbaum, J. N. Animal models of human amyloidoses: are transgenic mice worth the time and trouble? FEBS letters. 583, 2663-2673 (2009).

3. Hall, A. M., Roberson, E. D. Mouse models of Alzheimer's disease. Brain Res Bull. 88, 3-12 (2012).

4. Westermark, G. T., Johnson, K. H., Westermark, P. Staining methods for identification of amyloid in tissue. Methods in enzymology. 309, 3-25 (1999).

5. Klingstedt, T., et al. Synthesis of a library of oligothiophenes and their utilization as fluorescent ligands for spectral assignment of protein aggregates. Org Biomol Chem. 9, 8356-8370 (2011).

6. Klingstedt, T., et al. Luminescent conjugated oligothiophenes for sensitive fluorescent assignment of protein inclusion bodies. Chembiochem. 14, 607-616 (2013).

7. Sjolander, D., et al. Establishing the fluorescent amyloid ligand h-FTAA for studying human tissues with systemic and localized amyloid. Amyloid. 23, 98-108 (2016).

8. Magnusson, K., et al. Multimodal fluorescence microscopy of prion strain specific PrP deposits stained by thiophene-based amyloid ligands. Prion. 8, 319-329 (2014).

9. Radde, R., et al. Abeta42-driven cerebral amyloidosis in transgenic mice reveals early and robust pathology. EMBO Rep. 7, 940-946 (2006).

10. Sturchler-Pierrat, C., et al. Two amyloid precursor protein transgenic mouse models with Alzheimer disease-like pathology. Proceedings of the National Academy of Sciences of the United States of America. 94, 13287-13292 (1997).

11. Nystrom, S., et al. Evidence for age-dependent in vivo conformational rearrangement within Abeta amyloid deposits. ACS chemical biology. 8, 1128-1133 (2013).

12. Aslund, A., et al. Novel pentameric thiophene derivatives for in vitro and in vivo optical imaging of a plethora of protein aggregates in cerebral amyloidoses. ACS chemical biology. 4, 673-684 (2009).

13. Nilsson, K. P., Herland, A., Hammarstrom, P., Inganas, O. Conjugated polyelectrolytes: conformation-sensitive optical probes for detection of amyloid fibril formation. Biochemistry. 44, 3718-3724 (2005).

14. Mahajan, V., et al. Cross beta-sheet conformation of keratin 8 is a specific feature of Mallory-Denk bodies compared with other hepatocyte inclusions. Gastroenterology. 141, 1080-1090 e1081-1087 (2011).

15. Sjolander, D., Bijzet, J., Hazenberg, B. P., Nilsson, K. P., Hammarstrom, P. Sensitive and rapid assessment of amyloid by oligothiophene fluorescence in subcutaneous fat tissue. Amyloid. 22, 19-25 (2015).

16. Arja, K., et al. Enhanced fluorescent assignment of protein aggregates by an oligothiophene-porphyrin-based amyloid ligand. Macromol Rapid Commun. 34, 723-730 (2013).

17. Psonka-Antonczyk, K. M., et al. Nanoscale Structure and Spectroscopic Probing of Abeta1-40 Fibril Bundle Formation. Front Chem. 4, 44 (2016).

18. Herrmann, U. S., et al. Structure-based drug design identifies polythiophenes as antiprion compounds. Sci Transl Med. 7, 299ra123 (2015).

19. Schutz, A. K., et al. The amyloid-Congo red interface at atomic resolution. Angewandte Chemie (International ed.) 50, 5956-5960 (2011).

20. Hammarstrom, P., et al. A fluorescent pentameric thiophene derivative detects in vitro-formed prefibrillar protein aggregates. Biochemistry. 49 6838-6845 (2010).

21. Final Report Summary - LUPAS (Luminescent polymers for in vivo imaging of amyloid signatures). European Commision, Community Research and Developement Information Service, 10 (2013).

22. Hahn, K., et al. Establishing and validating the fluorescent amyloid ligand h-FTAA (heptamer formyl thiophene acetic acid) to identify transthyretin amyloid deposits in carpal tunnel syndrome. Amyloid., 1-9 (2017).

23. Johansson, L. B., et al. An azide functionalized oligothiophene ligand--a versatile tool for multimodal detection of disease associated protein aggregates. Biosens Bioelectron. 63, 204-211 (2015).

24. Nordeman, P., et al. (11)C and (18)F Radiolabeling of Tetra- and Pentathiophenes as PET-Ligands for Amyloid Protein Aggregates. ACS Med Chem Lett. 7, 368-373 (2016). 